\title{
Neutrophil swarms require LTB4 and integrins at sites of cell death in vivo
}

\author{
Tim Lämmermann ${ }^{1}$, Philippe V. Afonso ${ }^{2}$, Bastian R. Angermann ${ }^{1}$, Ji Ming Wang ${ }^{3}$, Wolfgang Kastenmüller ${ }^{1,4}$, Carole A. Parent ${ }^{2}$ \\ \& Ronald N. Germain ${ }^{1}$
}

Neutrophil recruitment from blood to extravascular sites of sterile or infectious tissue damage is a hallmark of early innate immune responses, and the molecular events leading to cell exit from the bloodstream have been well defined ${ }^{1,2}$. Once outside the vessel, individual neutrophils often show extremely coordinated chemotaxis and cluster formation reminiscent of the swarming behaviour of insects $^{3-11}$. The molecular players that direct this response at the single-cell and population levels within the complexity of an inflamed tissue are unknown. Using two-photon intravital microscopy in mouse models of sterile injury and infection, we show a critical role for intercellular signal relay among neutrophils mediated by the lipid leukotriene B4, which acutely amplifies local cell death signals to enhance the radius of highly directed interstitial neutrophil recruitment. Integrin receptors are dispensable for long-distance migration $^{12}$, but have a previously unappreciated role in maintaining dense cellular clusters when congregating neutrophils rearrange the collagenous fibre network of the dermis to form a collagen-free zone at the wound centre. In this newly formed environment, integrins, in concert with neutrophil-derived leukotriene B4 and other chemoattractants, promote local neutrophil interaction while forming a tight wound seal. This wound seal has borders that cease to grow in kinetic concert with late recruitment of monocytes and macrophages at the edge of the displaced collagen fibres. Together, these data provide an initial molecular map of the factors that contribute to neutrophil swarming in the extravascular space of a damaged tissue. They reveal how local events are propagated over large-range distances, and how auto-signalling produces coordinated, self-organized neutrophil-swarming behaviour that isolates the wound or infectious site from surrounding viable tissue.

Neutrophil swarming has been observed using intravital microscopy in inflamed, infected or sterilely wounded tissues ${ }^{3-11,13}$, and a series of sequential phases have been described ${ }^{3,4}$ : (1) initial chemotaxis of individual neutrophils close to the damage, followed by (2) amplified chemotaxis of neutrophils from more distant interstitial regions, leading to (3) neutrophil clustering. To study the molecules controlling these distinct neutrophil-response phases, we used an inducible model of sterile skin injury in which a brief intense two-photon laser pulse causes focal, dermis-restricted tissue damage (Supplementary Fig. 2) ${ }^{4}$. We were specifically interested in how neutrophils coordinate swarming in the extravascular space, so we performed two-photon intravital microscopy (2P-IVM) of neutrophils that had already exited blood vessels and entered a mildly inflamed dermis before laser damage. Focal injury induced substantial interstitial chemotaxis of lysozyme 2-green fluorescent protein (Lyz2-GFP, also known as LysM-GFP)positive neutrophils/monocytes that lasted $\sim 25-40 \mathrm{~min}$ before cells accumulated in a cluster at the damage site and recruitment stopped (Fig. 1a). The dynamic behaviour of neutrophils differed from CX3CR1positive macrophages/monocytes in the same environment, with neutrophils immediately showing highly directed chemotaxis towards the wound centre at high speeds $\left(10-20 \mu \mathrm{m} \mathrm{min}^{-1}\right)$ and the CX3CR1positive cells migrating at slower speeds $\left(3-5 \mu \mathrm{m} \mathrm{min}^{-1}\right)$ and undergoing

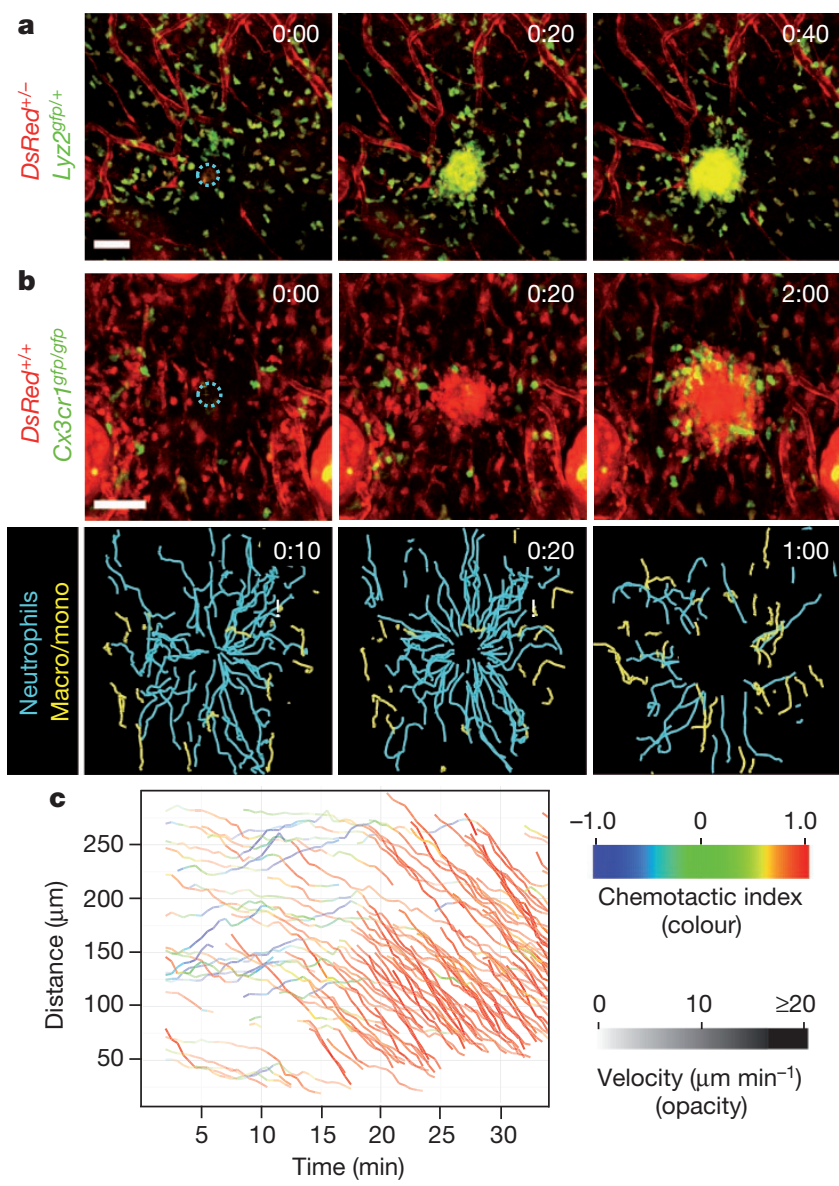

Figure 1 Neutrophil extravascular swarming dynamics. 2P-IVM on intact ear dermis of anaesthetized mice. Interstitial cell recruitment towards focal damage (blue dotted circle) was recorded. a, b, Time-lapse sequence of endogenous innate immune cell dynamics in $D s \operatorname{Red}^{+/-} \mathrm{Lyz}^{\mathrm{gfp/+}} \mathrm{Tyr} \mathrm{r}^{\mathrm{c}-2 J / c-2 J}$ mice (myelomonocytic cells in green-yellow, stroma in red) (a) and DsRed ${ }^{+/+} \mathrm{C} \times 3 \mathrm{cr} 1^{g f p / g f p} \mathrm{Tyr}^{c-2 J / c-2 J}$ mice (macrophages/monocytes in green, neutrophils and stroma in red) (b, top). Cell tracks over the last $10 \mathrm{~min}(n=4)$ (b, bottom). Scale bars, $50 \mu \mathrm{m}$. Time, h:min. c, Distance-time plot (DTP) of intradermal (i.d.) injected bone marrow neutrophils; individual cell-migration paths towards the damage site are each highlighted with instantaneous chemotactic index (colour) and velocity (opacity). Representative experiment of $n=169$. 
a chemotactic response only after an initial neutrophil cluster had formed. In contrast to neutrophils, macrophages/monocytes never entered the developing cell cluster, but assembled around it during cessation of the neutrophil swarm growth (Fig. 1b, Supplementary Fig. 3a and Supplementary Video 1). As reported previously, the early dynamics of endogenous neutrophils were biphasic (Supplementary Fig. 3b) and similar for neutrophils that were isolated from mouse bone marrow (Fig. 1c and Supplementary Video 2) or human peripheral blood (Supplementary Fig. 3c) and then injected into the dermis. In $79 \%$ of the experiments (169 out of 213 ), a first phase (1-15 min) of initial neutrophil chemotaxis close to the damage was followed by a dramatic second phase of substantial neutrophil recruitment from distant sites involving markedly increased directionality and speed (Fig. 1c). We noticed the appearance of cell fragments around developing neutrophil clusters, suggesting that cell death could lead to release of components driving neutrophil swarming ${ }^{14}$. Using propidium iodide, we detected a clear kinetic correlation between the death of a few neutrophils at the damage site and the onset of the amplified second phase of neutrophil recruitment (Fig. 2a, Supplementary Figs 4 and $5 \mathrm{a}$ and Supplementary Video 3). Most of the neutrophils in the developing cell cluster remained viable (Supplementary Fig. 5b), suggesting that the death of only a small number of neutrophils was sufficient to drive the substantial chemotactic response of the neutrophil population.

Although neutrophil death appeared to serve as a catalyst for swarming, it was unclear how such a signal would propagate through the structurally dense tissue in just a few minutes to initiate acute chemotaxis of neutrophils at sites more than $300 \mu \mathrm{m}$ away from the core lesion, and also maintain directional migration for $\sim 25-40 \mathrm{~min}$. To investigate the nature of this signal, we performed intradermal co-injection experiments with control and gene-deficient neutrophils and imaged them side by side in the same tissue volume in each experiment. The experimental set-up eliminated problematic quantitative comparisons based on measurements of wild-type and mutant cells imaged in different experiments that can be influenced by varying tissue composition in the imaging volume (Supplementary Fig. 6). More importantly, this protocol bypassed the extravasation step, which allowed for the study of neutrophils depleted of molecules essential for vessel exit.

Neutrophils express more than 30 cell-surface receptors for various attractants, which upon activation and signalling rearrange the cytoskeleton to yield a functionally polarized neutrophil that is poised for directed migration ${ }^{15,16}$. In these cells, most G-protein-coupled receptors (GPCRs) act through the predominantly expressed $\mathrm{G} \alpha_{\mathrm{i}}$ isoforms $\mathrm{G} \alpha_{\mathrm{i} 2}$ and $\mathrm{G} \alpha_{\mathrm{i} 3}$. We found that only neutrophils genetically lacking $\mathrm{G} \alpha_{\mathrm{i} 2}$ $\left(\right.$ Gnai2 $\left.^{-l-}\right)$, but not $\mathrm{G} \alpha_{\mathrm{i} 3}\left(\right.$ Gnai3 $\left.^{-l-}\right)$, show impaired interstitial chemotaxis (Supplementary Fig. 7) ${ }^{17}$. As $\mathrm{G} \alpha_{\mathrm{i} 2}$ couples to several neutrophil GPCRs, we next investigated the function of individual GPCRs. Most knockouts for single receptor genes tested (Fpr1, Fpr2, Cxcr2, Supplementary Fig. 8 and Supplementary Video 4; C5ar1, Ccr1, Ccr2, Ccr5, Cxcr6, Ptafr, P2ry2, data not shown)-many of which have been previously reported to have important roles in inflammatory, infectious or autoimmune conditions (Supplementary Information)showed normal interstitial chemotaxis, indicating that their respective ligands are not uniquely involved in the recruitment phase of the swarming response. Similarly, migration of neutrophils lacking receptors that detect the presence of danger signals (Myd88, Il1r1, Tnfrsf1a and Il1r1, P2rx7) was normal (data not shown). However, neutrophils lacking the high-affinity receptor for leukotriene B4 (LTB4) showed a uniquely impaired swarming response. During early phases ( $<15 \mathrm{~min}$ ), Ltb $4 r 1^{-1-}$ neutrophils close to the damage site still performed chemotaxis towards the wound, whereas cells from more distant sites were only poorly recruited (Fig. 2b, Supplementary Fig. 9a and Supplementary Video 5), a phenotype that became even more striking when a
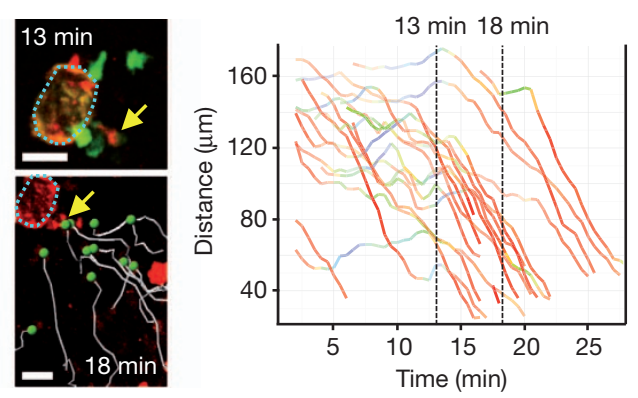

b

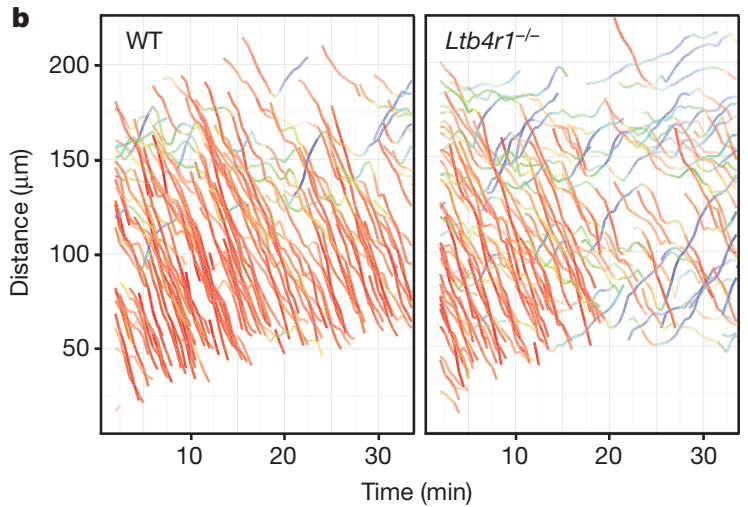

Figure $2 \mid$ LTB4 promotes neutrophil recruitment from distant sites. a, 2PIVM images of a single neutrophil becoming propidium iodide-positive (arrow) at $13 \mathrm{~min}$ and its correlation to neutrophil-amplified chemotaxis (white tracks). DTP analysis for migration paths coloured as in Fig. 1. b, Comparative analysis of interstitial recruitment after i.d. co-injection of $L t b 4 r 1^{-/-}$and wildtype (WT) neutrophils into $\mathrm{Tyr}^{c-2 J / c-2 J}$ mice. DTP of one representative experiment $(n=8)$. c, Time-course of migration tracks towards $10-\mu \mathrm{m}$
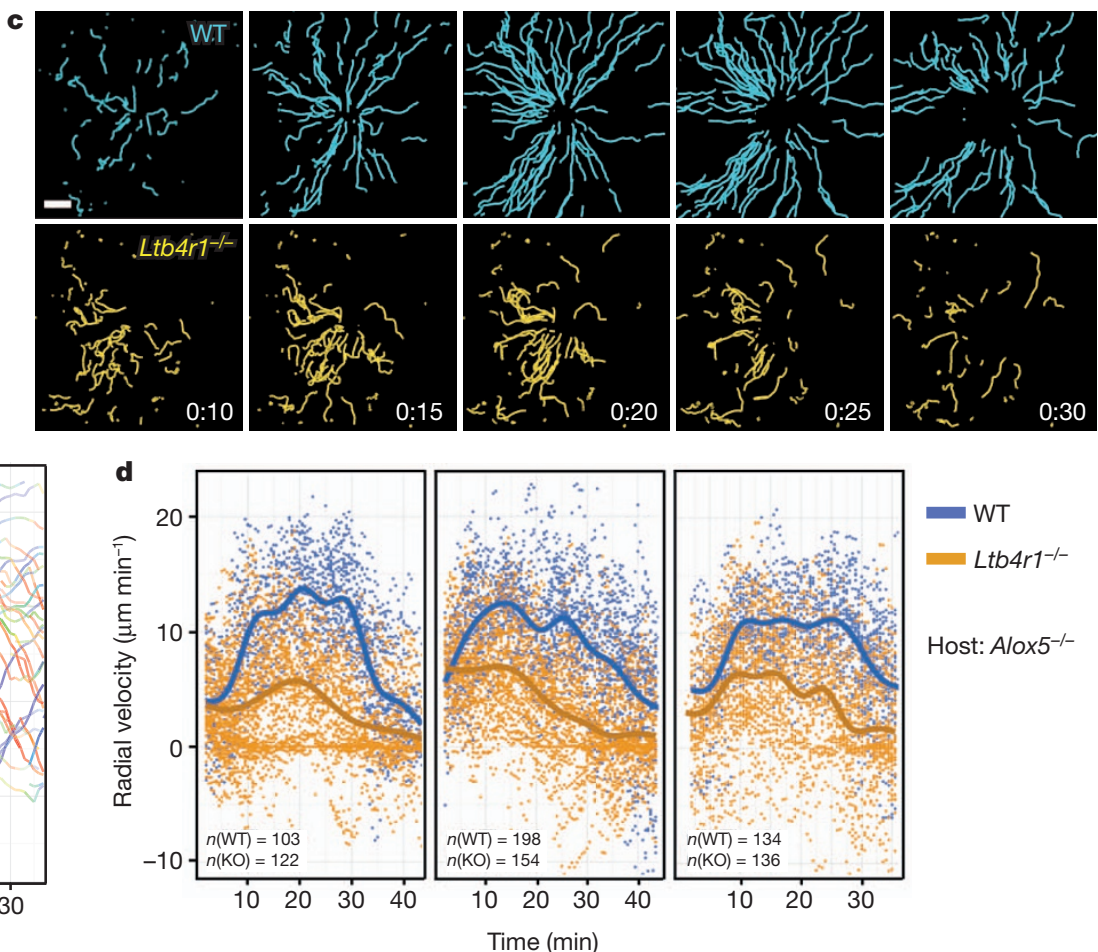

damage. Time, h:min. Scale bars, $20 \mu \mathrm{m}$ (a), $50 \mu \mathrm{m}$ (c). Track durations, $5 \mathrm{~min}$ (a), $10 \mathrm{~min}$ (c). d, Comparative analysis of interstitial recruitment after i.d. coinjection of $L t b 4 r 1^{-1-}$ knockout (KO) and wild-type neutrophils into Alox $5^{-1-} \mathrm{Tyr}^{c-2 J / c-2 J}$ mice. Radial velocity-time plots with regression lines showing the recruitment dynamics for three individual experiments ( of $n=7$ ). $n$ (in graph) indicates the number of analysed tracks. Each dot represents the instantaneous radial velocity for one cell at that time point. 

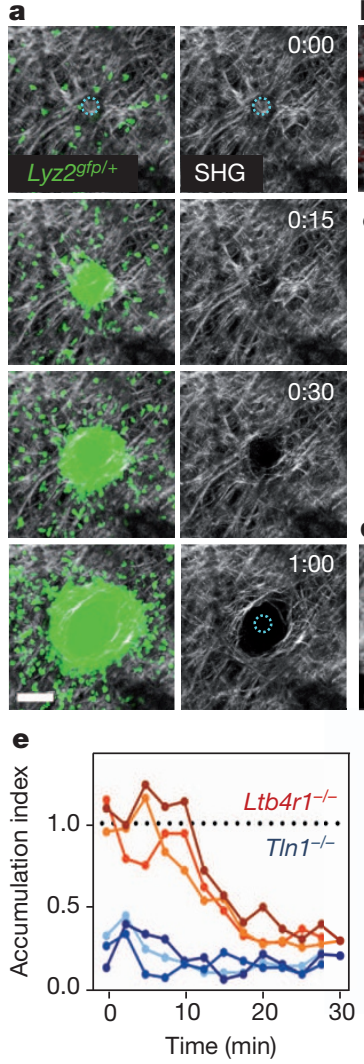

b

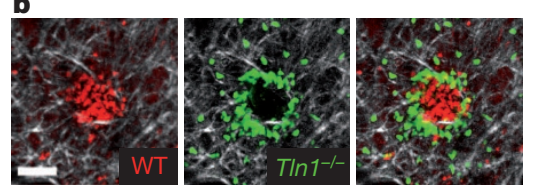

$0: 15$
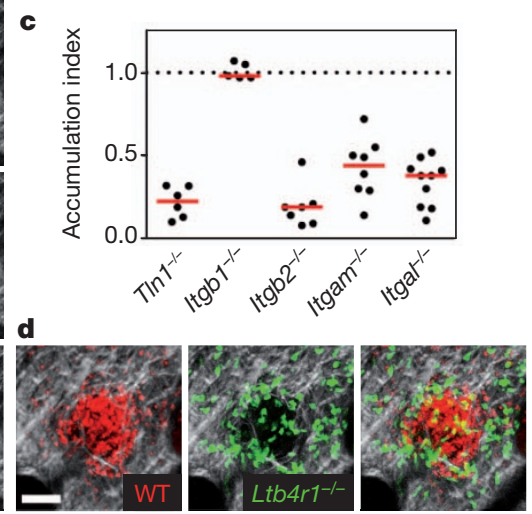

f

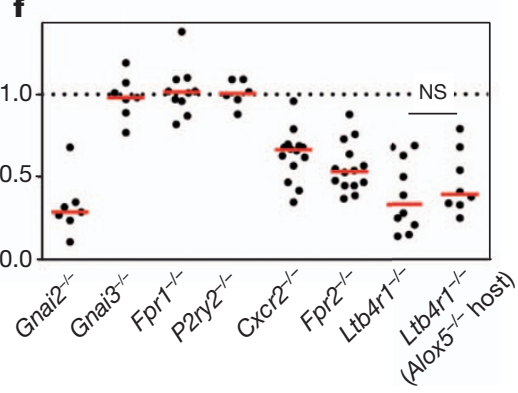

Figure $3 \mid$ Integrin and GPCR signalling at the neutrophil cluster. a, After focal damage (blue dotted circle), congregating neutrophils rearrange collagenous fibres (visualized by collagen second harmonic generation, SHG). Time, h:min. Scale bar, $50 \mu \mathrm{m}$. b-f, Comparative analysis of neutrophil clustering after i.d. co-injection of gene-deficient and wild-type neutrophils into Tyr ${ }^{c-2 J / c-2 J}$ or Alox $5^{-1-} T y r^{c-2 J / c-2 J}$ mice. b, d, 2P-IVM images at the end point of the clustering response. Scale bars, $50 \mu \mathrm{m}$. c, f, Accumulation index as a quantitative parameter for neutrophil entry into the collagen-free wound centre. Each dot represents analysis of one damage site. Median in red. NS, nonsignificant (Mann-Whitney $U$-test). e, Time-course of neutrophil accumulation in the wound centre. Three representative experiments are presented for each gene deficiency.

the damage size was smaller (Fig. 2c, Supplementary Fig. 10 and Supplementary Video 6). This reduction in neutrophil recruitment was mainly due to impaired chemotaxis rather than chemokinesis, and was most obvious during the late phases of the swarming response (>15 min) (Supplementary Fig. 9b).

In inflamed tissues, LTB4 can originate from several cellular sources. Earlier studies implicated a role for neutrophil-derived LTB4 in the initial stages of neutrophil recruitment from blood into inflamed tissues and disease progression ${ }^{18-22}$. To examine whether neutrophils autoamplified the swarming response through LTB4 production and signalling, we performed injection studies into 5-lipoxygenase-deficient mice $\left(\right.$ Alox $\left.5^{-/-}\right)$that cannot synthesize leukotrienes, such that only injected neutrophils could act as an LTB4 source. When co-injected with control cells, $L t b 4 r 1^{-1-}$ neutrophils again showed impaired interstitial chemotaxis and recruitment from distant sites (Fig. 2d, Supplementary Fig. 11 and Supplementary Video 7). When LTB4-secreting wild-type neutrophils were injected alone, they migrated to the injury from distances $>200 \mu \mathrm{m}$ and formed large clusters. By contrast, when we injected Alo $\times 5^{-1-}$ neutrophils alone into Alo $\times 5^{-1-}$ hosts so that no leukotrienes were present, only neutrophils close to the damage site $(<100 \mu \mathrm{m})$ were transiently recruited, resulting in small neutrophil clusters (Supplementary Fig. 12). Although these experiments establish an important role for neutrophil-derived LTB4 in recruiting neutrophils from distant sites in a feed-forward manner, they also show the existence of short-range chemotactic signals from the initial tissue injury and/or locally dying cells, as even in the absence of leukotrienes small clusters can form. We conclude that several of these initial primary factors not only act as short-range chemotactic signals, but also induce LTB4 secretion in neutrophils to acutely enhance the radius of neutrophil recruitment (Supplementary Fig. 1, panels 1-4), consistent with previous data on multiple LTB4-inducing factors ${ }^{22-25}$. This model is in agreement with earlier in vitro studies showing that primary signals induced secretion of LTB4 that acts as a signal relay molecule to improve chemotaxis of a whole neutrophil population ${ }^{23}$.

Because LTB4 not only induces intracellular polarity to direct chemotaxis, but can also regulate neutrophil adhesiveness by activating integrin receptors ${ }^{26,27}$, we next targeted integrin functionality by injecting neutrophils deficient for talin $\left(T \ln 1^{-{ }^{-}}\right)$. Talin interaction with integrin cytoplasmic domains is crucial for integrin activation, substrate binding and coupling of filamentous actin to adhesion sites ${ }^{28}$. Because interstitial chemotaxis of $T \ln 1^{-1-}$ cells was unimpaired (Supplementary Fig. 13 and Supplementary Video 8), high-affinity integrin function was dispensable for neutrophil interstitial migration in vivo, thus confirming earlier studies with dendritic cells in skin explants $^{12}$. Together, our results demonstrate that LTB4 has a nonredundant role in directing tissue-migrating neutrophils to sites of tissue injury by activating chemotaxis signal pathways.

We next sought to analyse the subsequent step of swarming when neutrophils accumulate and form substantial cell clusters. Visualization of the dense dermal collagen network using the second harmonic generation signal showed clearance of visible fibres from the core of the wound where the densest clustering occurred (Fig. 3a), an effect that was not due to thermal damage (Supplementary Fig. 14a, b). Congregating neutrophils appeared to physically exclude collagen fibres from the core of the cell infiltrate (Supplementary Video 9), although some limited proteolysis of extracellular matrix structures cannot be ruled out. Notably, monocytes always aligned along collagen fibres at the outer edges of the cell cluster, suggesting that neutrophils are specifically adapted to enter the collagen-free centre (Supplementary Fig. 14c). When we visualized both endogenous neutrophils and the cellular actin cortex of injected Lifeact-GFP ${ }^{+/-}$neutrophils in the collagenfree wound core, we could clearly observe neutrophils migrating in contact with each other with motion paths and focal filamentous actin accumulations that were suggestive of adhesive interactions (Supplementary Video 10$)$. Indeed, when $T \ln 1^{-1-}$ or $\operatorname{Itgb2} 2^{-1-}$ ( $\beta 2$ integrindeficient) neutrophils were co-injected with control cells, they showed a striking phenotype, as they were completely unable to enter the collagen-free zone and accumulated at the edge of the wild-type neutrophil cluster (Fig. 3b, c and Supplementary Videos 11, 12). Deficiency in either LFA-1 ( Itgal $\left.^{-1-}\right)$ or Mac-1 $\left(\right.$ Itgam $\left.^{-1-}\right)$ had a measurable effect on central accumulation, suggesting that both were involved in cell adhesion and movement within the collagen-free zone (Fig. $3 \mathrm{c}$ and Supplementary Fig. 15a). These unexpected findings indicate that high-affinity integrins are critical for neutrophil accumulation in the collagen-free wound centre, which fundamentally differs in its extracellular architecture from the surrounding intact dermal interstitium where leukocyte migration does not require high-affinity integrin function.

We next examined the role of GPCR signals in regulating neutrophil aggregation at the wound. Gnai $2^{-/-}$neutrophils were excluded from the central neutrophil cluster over time, whereas Gnai3 $^{-1-}$ accumulated normally (Supplementary Fig. 15b and Supplementary Video 13). When investigating individual GPCRs, we detected impaired aggregation for neutrophils lacking CXCR2, FPR2 or LTB4R1, with the latter showing the strongest impact on the aggregation response (Fig. 3d, f and Supplementary Video 14; other tested GPCR or receptors had no effect, Supplementary Fig. 15). In contrast to adhesion-deficient $T \ln 1^{-1-}$ cells, $\operatorname{Ltb} 4 r 1^{-1-}$ neutrophils were intermixed with wild-type 
cells at the earliest aggregation stages. Over time, however, $L t b 4 r 1^{-1-}$ neutrophils were excluded from the growing cluster (Fig. 3e and Supplementary Fig. 16a). Similar results were obtained when $L t b 4 r 1^{-1-}$ and wild-type neutrophils were injected into Alox $5^{-1-}$ hosts (Fig. $3 \mathrm{f}$ and Supplementary Video 14), indicating that neutrophils secrete LTB4 to regulate clustering in a feed-forward manner. Detection of ligands for other cluster-mediating receptors, such as CXCL2 for CXCR2 and CRAMP for FPR2, in accumulating neutrophils (Supplementary Fig. 16b) suggests that signalling through multiple GPCR, especially LTB4R1, may act to increase neutrophil aggregation to maintain tight association and motility for continued uptake of cellular wound debris or potential pathogens in the cellular cluster (Supplementary Fig. 1, panels 5-8).

Our findings thus identify a crucial dual function for LTB4 in both the recruitment and the clustering phases of the swarm response to sterile injury. To test the relevance of these findings in an infectious situation, we investigated the role of LTB4 during transient neutrophil swarming in infected lymph nodes ${ }^{3}$. We have shown previously that Pseudomonas aeruginosa induces cell death of subcapsular macrophages, which subsequently leads to neutrophil recruitment to the lymph node ${ }^{29}$. Whereas endogenous neutrophils in control mice formed several large but transient cell clusters and were highly chemotactic between the competing transient clusters, $L t b 4 r 1^{-1-}$ neutrophils

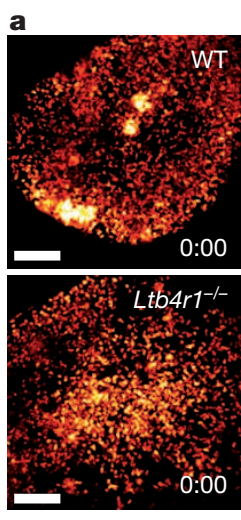

b

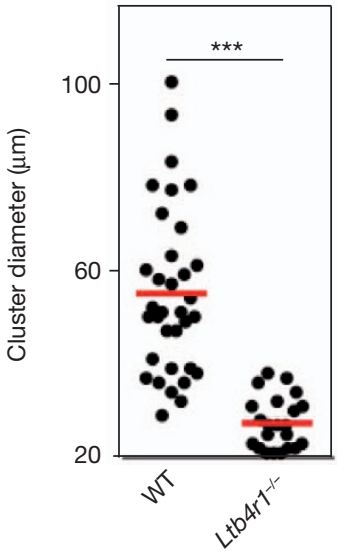

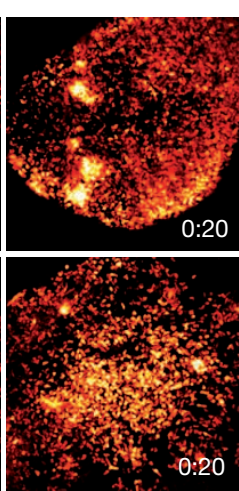

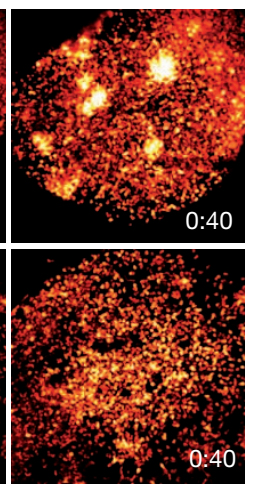

c

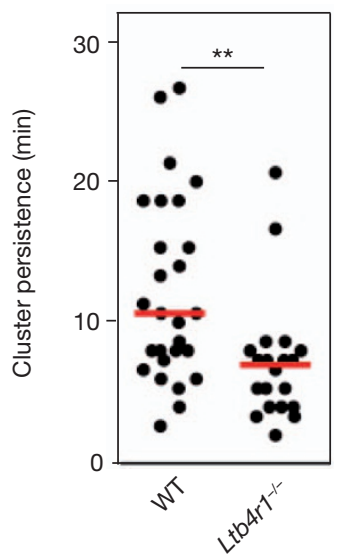

Figure $4 \mid$ LTB4 requirement for swarming in infected lymph nodes. Mice were infected with $P$. aeruginosa-GFP in the footpad and 2P-IVM was then performed on the draining popliteal lymph nodes at the times indicated. a, Time-lapse sequence of infected subcapsular sinuses of wild-type $L y z 2^{g f p /+}$ (top) and $L t b 4 r 1^{-/-} L y z 2^{g f p /+}$ (bottom) mice. Analysis was performed when comparable neutrophil numbers were in the sinus (wild type, $3 \mathrm{~h} ; \mathrm{Ltb} 4 \mathrm{r} \mathrm{1}^{-1-}$, $4.5 \mathrm{~h}$ ). Neutrophil-GFP signal is pseudo-coloured (heat map) to indicate neutrophil clusters (white). Time, h:min. Scale bars, $100 \mu \mathrm{m}$.

$\mathbf{b}, \mathbf{c}$, Quantification of cluster diameter (b) and persistence (c). Data pooled from three independent experiments. $\mathbf{b}, * * * P<0.001$ (Student's $t$-test); c, ${ }^{*} P<0.01$ (Mann-Whitney $U$-test). Red bars, mean (b), median (c). were slower and formed only very small, if any, clusters (Fig. 4a and Supplementary Video 15). Consequently, neutrophil cluster diameter and persistence were significantly lower in the absence of LTB4 signalling (Fig. 4b, c), thus confirming the results of the controlled inducible model of focal laser skin injury and extending them to other tissues, forms of cell death and infectious conditions.

The chemoattractants that direct the neutrophil tissue response can stem from the injured tissue, resident cells, recruited blood-derived leukocytes and potential pathogens. The specific mixture of these various signals will determine the neutrophil chemotactic response at each inflammatory or infectious site. In large sterile liver injuries, integrin-dependent intravascular neutrophil migration requires CXCR2 ligands on liver sinusoids and formyl peptides in the injury zone ${ }^{13}$. Here we have investigated extravascular neutrophil swarming at very small focal sites of sterile tissue injury in the skin $(\sim 1,000 \times$ smaller than those examined in the liver model $)^{4}$ and in infected lymph nodes ${ }^{3}$. We found that local cell death initiates dramatic swarm-like interstitial neutrophil recruitment and clustering, with a key role for LTB4 as a unique intercellular communication signal between neutrophils that allows rapid integrin-independent neutrophil recruitment through the tissue. Such insights should prove useful for studies in which local sterile or pathogen-induced cell death characterizes innate immune cell dynamics $^{3-11}$, and the molecules identified may serve as potential targets for therapeutic intervention in destructive neutrophil-dependent inflammatory processes.

\section{METHODS SUMMARY}

Mice. Supplementary Information lists all mouse strains used in this study. All mice were maintained in specific-pathogen-free conditions at an Association for Assessment and Accreditation of Laboratory Animal Care-accredited animal facility at the NIAID and were used under a study protocol approved by NIAID Animal Care and Use Committee (National Institutes of Health).

2P-IVM and focal tissue damage. 2P-IVM of ear pinnae of anaesthetized mice was performed as previously described ${ }^{5}$. For the study of endogenous innate immune cells in the extravascular space, anaesthetized transgenic reporter mice underwent a 15-s skin trauma to recruit neutrophils from the circulation to the dermal interstitium, 3-4h before focal tissue damage was induced. For genefunction experiments, neutrophils were isolated from bone marrow of control and gene-deficient mice, differentially dye-labelled, and injected intradermally at a 1:1 ratio into $T y r^{c-2 J / c-2 J}$ mice, $2-3 \mathrm{~h}$ before focal tissue damage was induced. Focal tissue damage by a brief two-photon laser pulse $(80 \mathrm{~mW})$ has been described previously ${ }^{4}$. All imaged mice were on the $T y r^{c-2 J / c-2 J}$ (B6.Albino) background to avoid laser-induced cell death of light-sensitive skin melanophages.

Image analysis. Three-dimensional object tracking using Imaris (Bitplane) retrieved cell spatial coordinates over time. These data were further processed using routines developed in the open source programming language $\mathrm{R}$ to retrieve dynamic parameters over time for individual cells (distance-time plot) and cell populations (radial velocity-time plot). For details on the source code and definition of chemotactic index, radial velocity and accumulation index, see Supplementary Information. Student's $t$-tests were performed after data were confirmed to fulfil the criteria of normal distribution and equal variance, otherwise MannWhitney $U$-tests were applied. Analyses were performed with GraphPad Prism 5 software.

Full Methods and any associated references are available in the online version of the paper.

Received 7 November 2012; accepted 11 April 2013.

Published online 26 May 2013.

1. Nathan, C. Points of control in inflammation. Nature 420, 846-852 (2002).

2. Ley, K., Laudanna, C., Cybulsky, M. I. \& Nourshargh, S. Getting to the site of inflammation: the leukocyte adhesion cascade updated. Nature Rev. Immunol. 7, 678-689 (2007).

3. Chtanova, T. et al. Dynamics of neutrophil migration in lymph nodes during infection. Immunity 29, 487-496 (2008).

4. Ng, L. G. et al. Visualizing the neutrophil response to sterile tissue injury in mouse dermis reveals a three-phase cascade of events. J. Invest. Dermatol. 131, 2058-2068 (2011).

5. Peters, N. C. et al. In vivo imaging reveals an essential role for neutrophils in leishmaniasis transmitted by sand flies. Science 321, 970-974 (2008). 
6. Bruns, S. etal. Production of extracellular traps against Aspergillus fumigatus in vitro and in infected lung tissue is dependent on invading neutrophils and influenced by hydrophobin RodA. PLoS Pathog. 6, e1000873 (2010)

7. Yipp, B. G. et al. Infection-induced NETosis is a dynamic process involving neutrophil multitasking in vivo. Nature Med. 81, 1386-1393 (2012).

8. Liese, J., Rooijakkers, S. H., van Strijp, J. A., Novick, R. P. \& Dustin, M. L. Intravital two-photon microscopy of host-pathogen interactions in a mouse model of Staphylococcus aureus skin abscess formation. Cell Microbiol. http://dx.doi.org/ 10.1111/cmi.12085 (2012)

9. Harvie, E. A., Green, J. M., Neely, M. N. \& Huttenlocher, A. Innate immune response to Streptococcus iniae infection in zebrafish larvae. Infect. Immun. 81, 110-121 (2013).

10. Kreisel, D. et al. In vivo two-photon imaging reveals monocyte-dependent neutrophil extravasation during pulmonary inflammation. Proc. Natl Acad. Sci. USA 107, 18073-18078 (2010).

11. Nakasone, E. S. et al. Imaging tumor-stroma interactions during chemotherapy reveals contributions of the microenvironment to resistance. Cancer Cel/ 21, 488-503 (2012).

12. Lämmermann, T. etal. Rapid leukocyte migration by integrin-independent flowing and squeezing. Nature 453, 51-55 (2008)

13. McDonald, B. et al. Intravascular danger signals guide neutrophils to sites of sterile inflammation. Science 330, 362-366 (2010)

14. Guggenberger, C., Wolz, C., Morrissey, J. A. \& Heesemann, J. Two distinct coagulase-dependent barriers protect Staphylococcus aureus from neutrophils in a three dimensional in vitro infection model. PLoS Pathog. 8, e1002434 (2012).

15. McDonald, B. \& Kubes, P. Cellular and molecular choreography of neutrophil recruitment to sites of sterile inflammation. J. Mol. Med. 89, 1079-1088 (2011)

16. Sánchez-Madrid, F. \& del Pozo, M. A. Leukocyte polarization in cell migration and immune interactions. EMBO J. 18, 501-511 (1999).

17. Cho, $\mathrm{H}$ etal. The loss of RGS protein-G $\alpha_{i 2}$ interactions results in markedly impaired mouse neutrophil trafficking to inflammatory sites. Mol. Cell. Biol. 32, 4561-4571 (2012).

18. Kim, N. D., Chou, R. C., Seung, E., Tager, A. M. \& Luster, A. D. A unique requirement for the leukotriene B4 receptor BLT1 for neutrophil recruitment in inflammatory arthritis. J. Exp. Med. 203, 829-835 (2006).

19. Chen, M. et al. Neutrophil-derived leukotriene B4 is required for inflammatory arthritis. J. Exp. Med. 203, 837-842 (2006)

20. Oyoshi, M. K. et al. Leukotriene B4-driven neutrophil recruitment to the skin is essential for allergic skin inflammation. Immunity 37, 747-758 (2012).

21. Chou, R. C. et al. Lipid-cytokine-chemokine cascade drives neutrophil recruitment in a murine model of inflammatory arthritis. Immunity 33, 266-278 (2010).

22. Sadik, C. D., Kim, N. D., Iwakura, Y. \& Luster, A. D. Neutrophils orchestrate their own recruitment in murine arthritis through $\mathrm{C} 5 \mathrm{aR}$ and $\mathrm{Fc} \gamma \mathrm{R}$ signaling. Proc. Natl Acad. Sci. USA 109, E3177-E3185 (2012).
23. Afonso, P. V. et al. LTB4 is a signal-relay molecule during neutrophil chemotaxis. Dev. Cell 22, 1079-1091 (2012)

24. DiPersio, J. F., Billing, P., Williams, R. \& Gasson, J. C. Human granulocytemacrophage colony-stimulating factor and other cytokines prime human neutrophils for enhanced arachidonic acid release and leukotriene B4 synthesis. J. Immunol. 140, 4315-4322 (1988).

25. Malawista, S. E., de Boisfleury Chevance, A., van Damme, J. \& Serhan, C. N. Tonic inhibition of chemotaxis in human plasma. Proc. Natl Acad. Sci. USA 105, 17949-17954 (2008)

26. Palmblad, J. et al. Leukotriene B4 is a potent and stereospecific stimulator of neutrophil chemotaxis and adherence. Blood 58, 658-661 (1981).

27. Ford-Hutchinson, A. W., Bray, M. A., Doig, M. V., Shipley, M. E. \& Smith, M. J. Leukotriene $B$, a potent chemokinetic and aggregating substance released from polymorphonuclear leukocytes. Nature 286, 264-265 (1980).

28. Calderwood, D. A. \& Ginsberg, M. H. Talin forges the links between integrins and actin. Nature Cell Biol. 5, 694-697 (2003).

29. Kastenmüller, W., Torabi-Parizi, P., Subramanian, N., Lämmermann, T. \& Germain, R. N. A spatially-organized multicellular innate immune response in lymph nodes limits systemic pathogen spread. Cell 150, 1235-1248 (2012).

Supplementary Information is available in the online version of the paper.

Acknowledgements We thank L. Birnbaumer, R. Fässler, E. Tuomanen, P. M. Murphy, S. Akira, S. Monkley, D. Critchley, R. Wedlich-Söldner and M. Sixt for providing mice for this study, J.G. Egen and J. Tang for assistance with imaging, M. Parsek for providing P. aeruginosa-GFP, J.H. Kehrl, P.M. Murphy, R. Varma and members of the Germain laboratory for discussions. This work was supported by the Intramural Research Program of National Institute of Allergy and Infectious Diseases and National Cancer Institute, National Institutes of Health. T.L. was supported by a Human Frontiers Science Program Long-Term Fellowship. W.K. is presently a member of the Deutsche Forschungsgemeinschaft (DFG)-funded excellence cluster ImmunoSensation, Bonn, Germany.

Author Contributions T.L. and R.N.G. designed the experiments, interpreted the data and wrote the paper. P.V.A., J.M.W. and C.A.P. contributed to data interpretation and experimental design, as well as the development of the final version of the paper. T.L. performed all skin-imaging experiments. W.K. performed intravital imaging of infected lymph nodes. B.R.A. developed the software for graphical display of imaging data. T.L., B.R.A. and P.V.A. conducted quantitative analysis of the data.

Author Information Reprints and permissions information is available at www.nature.com/reprints. The authors declare no competing financial interests. Readers are welcome to comment on the online version of the paper. Correspondence and requests for materials should be addressed to T.

(laemmermannt@niaid.nih.gov) or R.N.G. (rgermain@niaid.nih.gov). 


\section{METHODS}

Mice. Supplementary Table 1 lists all mouse strains and crosses used in this study. Gnai2 $^{-/-30}$, Gnai3 $^{-/-31}, \operatorname{Itgbl}^{\mathrm{fl} / \mathrm{fl} 32}, \operatorname{Tln} 1^{\mathrm{fl} / \mathrm{fl} 33}, \mathrm{Ccrl}^{-/-34}, \mathrm{Fprl}^{-/-35}, \mathrm{Fpr}^{-/-36}$, Ptafr $^{-1-37}, M y d 88^{-1-38}$ and Lifeact-GFP ${ }^{+/-39}$ mice have been described elsewhere. $L y z 2^{g f p / g f p 40}$ and $I l 1 r 1^{-/-41}$ mice were obtained from Taconic Laboratories through a special contract with the NIAID. All other mouse strains were purchased from Jackson Laboratories. All mice were maintained in specific-pathogen-free conditions at an Association for Assessment and Accreditation of Laboratory Animal Care-accredited animal facility at the NIAID and were used under a study protocol approved by NIAID Animal Care and Use Committee (National Institutes of Health).

2P-IVM of ear skin and infected lymph nodes. Two-photon intravital imaging of ear pinnae of anaesthetized mice was performed as previously described ${ }^{5,42}$ Mice were anaesthetized using isoflurane (Baxter; 2\% for induction, 1-1.5\% for maintenance, vaporized in an 80:20 mixture of oxygen and air) and placed in a lateral recumbent position on a custom imaging platform such that the ventral side of the ear pinna rested on a coverslip. A strip of Durapore tape was placed lightly over the ear pinna and affixed to the imaging platform to immobilize the tissue Care was taken to minimize pressure on the ear. Images were captured towards the anterior half of the ear pinna where hair follicles are sparse. Images were acquired using an inverted LSM 510 NLO multiphoton microscope (Carl Zeiss Microimaging) enclosed in a custom-built environmental chamber that was maintained at $32{ }^{\circ} \mathrm{C}$ using heated air. This system had been custom fitted with three external non-descanned photomultiplier tube detectors in the reflected light path. Images were acquired using a $25 \times / 0.8$ numerical aperture (NA) PlanApochromat objective (Carl Zeiss Imaging) with glycerol as immersion medium. Fluorescence excitation was provided by a Chameleon XR Ti:Sapphire laser (Coherent) tuned to $850 \mathrm{~nm}$ for dye excitation and generation of collagen second harmonic signal, or $920 \mathrm{~nm}$ for enhanced GFP (eGFP) excitation and $940 \mathrm{~nm}$ for excitation of both DsRed and eGFP. For four-dimensional data sets, threedimensional stacks were captured every $30 \mathrm{~s}$, unless otherwise specified. Al imaged mice were on the $\mathrm{Tyr}^{\mathrm{c}-2 J / c-2 J}$ (B6.Albino) background to avoid laser-induced cell death of light-sensitive skin melanophages ${ }^{43}$. 2 P-IVM on $P$. aeruginosa-infected lymph nodes was performed as previously described ${ }^{29} \cdot 10^{7}$ colony-forming units of GFP-expressing Pseudomonas aeruginosa ${ }^{44}$ (provided by M. Parsek) were diluted in PBS and injected in the footpad $(30 \mu \mathrm{l})$ of wild-type $L y z 2^{g f p /+}$ and $L t b 4 r 1^{-1-} L y z 2^{g f p /+}$ mice and draining lymph nodes imaged at indicated times after infection using a Zeiss 710 microscope equipped with a Chameleon laser (Coherent) and a $20 \times$ water-dipping lens (NA 1.0, Zeiss). The microscope was enclosed in an environmental chamber in which anaesthetized mice were warmed by heated air, and the surgically exposed lymph node was kept at $36-37^{\circ} \mathrm{C}$ with warmed PBS. For 2P-IVM of the subcapsular sinus, we used a $z$-stack of $40-50 \mu \mathrm{m}$, $3-\mu \mathrm{m}$ step size and acquired images every $40 \mathrm{~s}$. Raw imaging data were processed with Imaris (Bitplane) using a Gaussian filter for noise reduction. All images and movies are displayed as two-dimensional maximum-intensity projections of 10 30- $\mu$ m-thick $z$-stacks.

Endogenous mouse innate immune cells. For the study of endogenous innate immune cells in the extravascular space, transgenic reporter mice were crossed with $T y r^{c-2 J / c-2 J}$ (B6.Albino) mice to yield $L y z 2^{g f p /+} T y r^{c-2 J / c-2 J}$ (myelomonocytic cells), DsRed ${ }^{+/-} L y z 2^{g f p^{\prime+}} \mathrm{Tyr}^{c-2 J / c-2 J}$ (myelomonocytic cells, stroma) and $\mathrm{DsRed}^{+/+} \mathrm{CX} 3 \mathrm{crl}^{g f p / g f p} \mathrm{Tyr}^{c-2 J / c-2 J}$ (macrophages/monocytes, stroma and neutrophils) mice. These animals were anaesthetized with isoflurane and underwent a brief skin trauma to recruit neutrophils from the circulation to the dermal interstitium. The anaesthetized mouse was placed on a scale and $30 \mathrm{~N}$ per $\mathrm{cm}^{2}$ pressure was applied for 15-20s on the mouse ear with the investigator's thumb. As this method induced initial neutrophil extravasation that stopped after $2-3 \mathrm{~h}$, this method was superior to other tested common inflammatory treatments (for example, chemical skin irritation) that all lead to neutrophil recruitment from blood over longer time periods. Three hours after brief skin trauma, mice were prepared for skin imaging as described above and rested in the heated environmental chamber for 30-60 min before the first focal tissue damage was induced. Neutrophil isolation, labelling and i.d. injection. For i.d. injection experiments, mouse neutrophils were isolated from bone marrow using a three-layer Percoll gradient of $78 \%, 69 \%$ and $52 \%$ as previously described ${ }^{45}$. Neutrophils were collected at the $69-78 \%$ interface and were highly purified $(>98 \%)$ as indicated by Lyz2eGFP $^{\text {hi }}$ Ly6G ${ }^{\text {pos }}$ Ly6C ${ }^{\text {neg }}$ phenotype in flow cytometry ${ }^{46}$ (data not shown), with viability $>98 \%$ by trypan blue staining. Neutrophils were washed three times with washing buffer ( $1 \times$ Hank's balanced salt solution, 1\% FBS, 2 mMEDTA). If further labelled with cell dyes, neutrophils were incubated for $15 \mathrm{~min}$ with either $0.8 \mu \mathrm{M}$ cell tracker red (CMTPX) or $1 \mu \mathrm{M}$ cell tracker green (CMFDA) in $1 \times$ HBSS supplemented with $0.0002 \%$ (w/v) pluronic F-127 (all Life Technologies). Neutrophils were washed four times with washing buffer, before a 1:1 ratio of differentially labelled control and gene-deficient neutrophils (each $>2 \times 10^{6}$ cells) was taken up in $1 \times$ PBS at a volume of $15-30 \mu \mathrm{l}$. A volume of $5 \mu \mathrm{l}$ neutrophil suspension was injected intradermally with an insulin syringe (31.5 GA needle, BD Biosciences) into the ventral side of the mouse ear pinnae. Recipient mice were always on the $T y r^{c-2 J / c-2 J}$ (B6.Albino) background. Two hours after injection, mice were prepared for skin imaging as described above and rested in the heated environmental chamber for 30-60 min before the first focal tissue damage was induced. For isolation of talin-deficient neutrophils, $T \ln 1^{\mathrm{fl} / \mathrm{fl}}$ mice were intercrossed with $M \times 1-c r e^{+/-}$mice ${ }^{47}$ to yield $T \ln 1^{\mathrm{f} / \mathrm{fl}} \mathrm{M} \times 1-c r e^{+/-}$. In these mice, Cre expression in the haematopoietic system was induced by intraperitoneal injection of $250 \mu \mathrm{g}$ Poly(I):Poly(C) (Amersham Biosciences). Five days after knockout induction neutrophils were isolated from bone marrow with high efficiency of talin depletion ${ }^{12}$ For most injection experiments, we interchanged dyes to exclude unspecific effects and never observed differences in migration or accumulation phenotypes. Whereas cell tracker green labels neutrophils homogeneously, cell tracker red gives a polarized neutrophil staining over time. Cell tracker red also leaks over time from cells, resulting in background fluorescence in the tissue and some fluorescent resident, non-motile, elongated macrophages taking up the dye. For experiments with human neutrophils, heparinized whole blood was obtained by venipuncture from healthy donors. Blood samples were obtained from anonymous blood donors enrolled in the NIH Blood Bank research program. Human neutrophils were isolated with Lympholyte-poly Cell Separation Media (Cedarline) as previously described ${ }^{48}$ Residual erythrocytes were removed using ammonium-chloride-potassium lysis buffer (Lonza).

Focal tissue damage. A protocol for focal skin tissue damage by a focused twophoton laser pulse has been described previously ${ }^{43}$ and used with slight modifications. The Chameleon XR Ti:sapphire laser (Coherent) was tuned to $850 \mathrm{~nm}$ and the laser intensity adjusted to $80 \mathrm{~mW}$. At pixel dimensions of $0.14 \times 0.14 \mu \mathrm{m}$, a circular region of interest of $25-35 \mu \mathrm{m}$ in diameter (approximately $1-2 \times 10^{-6}$ $\mathrm{mm}^{3}$ in volume) (unless otherwise specified) was defined in one focal plane, followed by laser scanning at a pixel dwell time of $0.8 \mu$ s for $35-50$ iterations depending on the tissue depth of the imaging field of view. The damage was restricted to dermal layers only (Supplementary Fig. 2). Immediately after laserinduced tissue damage, imaging of the neutrophil response was started at typical voxel dimensions of $0.72 \times 0.72 \times 2 \mu \mathrm{m}$. We performed a maximum of three consecutive experiments per mouse ear.

Data analysis. Three-dimensional object tracking using Imaris (Bitplane) retrieved cell spatial coordinates $(x, y, z)$ over time. These data were further processed using routines composed in the open source programming language $\mathrm{R}$ (source code in Supplementary Material) to retrieve dynamic parameters for individual cells (distance-time plot, DTP) and cell populations (radial velocity-time plot). The chemotactic index was defined as $\cos (\alpha)$, with $\alpha$ as the angle between the distance vector to the damage site and the actual movement vector (Supplementary Fig. 4). The instantaneous radial velocity is the product of the chemotactic index and the cell's instantaneous velocity (migrated distance over $30 \mathrm{~s}$ ) Reconstructed cell tracks were filtered using a moving average with a width of $2.5 \mathrm{~min}$ to suppress spurious direction changes due to limited resolution of the tracking algorithm and small scale features such as other cells in the path of the migrating cell. Paths that had durations of less than 3 min were dropped. Velocities and the chemotactic index were calculated using central differences from the filtered paths. Plots including regression lines were generated using the ggplot2 package (version 0.8.9) for $\mathrm{R}$ (version 2.13.1).

The accumulation index as measure of cell entry into the collagen-free zone was defined as the ratio of fluorescent signal from gene-deficient cells in the collagenfree zone versus total signal at the wound site divided by the ratio of fluorescent signal from control cells in the collagen-free zone versus total signal at the wound site. Fluorescent signals from static 2P-IVM images were quantified in ImageJ software (National Institutes of Health).

Immunofluorescence in ear skin whole mounts. One to two hours after induction of several focal tissue damage sites, mice were euthanized, ears excised, divided into dorsal and ventral halves, ventral halves fixed in $1 \%$ paraformaldehyde (Electron Microscopy Sciences) and stained with antibodies diluted in washing buffer consisting of $1 \times$ PBS, 1\% BSA and 0.025\% (v/v) Triton X-100 (SigmaAldrich). For identification of neutrophils in $\operatorname{DsRed}^{+/+} \mathrm{Cx} \mathrm{cr} 1^{g f p / g f p} \mathrm{Tyr}^{\mathrm{c}-2 J / c-2 J}$ mice, ventral ear whole mounts were stained with Alexa Fluor 647 anti-mouse Ly-6G Antibody (clone 1A8, BioLegend) ${ }^{49}$. For detection of CXCL2 (MIP-2) and CRAMP in neutrophil clusters, anti-MIP2 antibody (R\&D Systems), anti-CRAMP antibody (Phoenix Pharmaceuticals), rabbit IgG isotype control and goat IgG isotype control (both Southern Biotech) were all conjugated to Alexa Fluor 555 according to the manufacturer's instructions (Life Technologies). Fixed ventral ear halves of $L y z 2^{g f p / g f p}$ mice were blocked with $10 \%$ normal goat serum or normal rabbit serum (both Southern Biotech) in washing buffer before staining with the Alexa Fluor 555-conjugated antibodies. All Alexa Fluor 555-conjugated antibodies gave strong punctate staining in the dermal tissue, but isotype controls never gave 
staining within neutrophils. Confocal microscopy on skin whole mounts was performed with a LSM 710 confocal microscope equipped with a $20 \times / 0.8 \mathrm{NA}$ Plan-Apochromat objective (Carl Zeiss Microimaging) using 1- $\mu \mathrm{m}$ optical slices. Statistical analysis. Student's $t$-tests were performed after data were confirmed to fulfil the criteria of normal distribution and equal variance; otherwise Mann-Whitney $U$-tests were applied. Analyses were performed with GraphPad Prism 5 software.

30. Rudolph, U. et al. Ulcerative colitis and adenocarcinoma of the colon in $\mathrm{G} \alpha_{\mathrm{i} 2^{-}}$ deficient mice. Nature Genet. 10, 143-150 (1995)

31. Pero, R. S. et al. $\mathrm{G} \alpha_{\mathrm{i} 2}$-mediated signaling events in the endothelium are involved in controlling leukocyte extravasation. Proc. Natl Acad. Sci. USA 104, 4371-4376 (2007).

32. Potocnik, A. J., Brakebusch, C. \& Fassler, R. Fetal and adult hematopoietic stem cells require beta1 integrin function for colonizing fetal liver, spleen, and bone marrow. Immunity 12, 653-663 (2000).

33. Petrich, B. G. et al. Talin is required for integrin-mediated platelet function in hemostasis and thrombosis. J. Exp. Med. 204, 3103-3111 (2007)

34. Gao, J. L. et al. Impaired host defense, hematopoiesis, granulomatous inflammation and type 1-type 2 cytokine balance in mice lacking CC chemokine receptor 1. J. Exp. Med. 185, 1959-1968 (1997).

35. Gao, J. L., Lee, E. J. \& Murphy, P. M. Impaired antibacterial host defense in mice lacking the $N$-formylpeptide receptor. J. Exp. Med. 189, 657-662 (1999).

36. Chen, K. et al. A critical role for the G protein-coupled receptor mFPR2 in airway inflammation and immune responses. J. Immunol. 184, 3331-3335 (2010).

37. Radin, J. N. et al. $\beta$-Arrestin 1 participates in platelet-activating factor receptormediated endocytosis of Streptococcus pneumoniae. Infect. Immun. $\mathbf{7 3}$, 7827-7835 (2005)
38. Adachi, O. et al. Targeted disruption of the MyD88 gene results in loss of IL-1- and IL-18-mediated function. Immunity 9, 143-150 (1998).

39. Riedl, J. et al. Lifeact mice for studying F-actin dynamics. Nature Methods 7 , 168-169 (2010)

40. Faust, N., Varas, F., Kelly, L. M., Heck, S. \& Graf, T. Insertion of enhanced green fluorescent protein into the lysozyme gene creates mice with green fluorescent granulocytes and macrophages. Blood 96, 719-726 (2000).

41. Glaccum, M. B. et al. Phenotypic and functional characterization of mice that lack the type I receptor for IL-1. J. Immunol. 159, 3364-3371 (1997).

42. Gaiser M. R et al Cancer-associated epithelial cell adhesion molecule (EpCAM; CD326) enables epidermal Langerhans cell motility and migration in vivo. Proc. Natl Acad. Sci. USA 109, E889-E897 (2012).

43. Li, J. L. et al. Intravital multiphoton imaging of immune responses in the mouse ear skin. Nature Protocols 7, 221-234 (2012).

44. Davies, D. G. et al. The involvement of cell-to-cell signals in the development of a bacterial biofilm. Science 280, 295-298 (1998).

45. Boxio, R., Bossenmeyer-Pourie, C., Steinckwich, N., Dournon, C. \& Nusse, O. Mouse bone marrow contains large numbers of functionally competent neutrophils. J. Leukoc. Biol. 75, 604-611 (2004).

46. Woodfin, A. et al. The junctional adhesion molecule JAM-C regulates polarized transendothelial migration of neutrophils in vivo. Nature Immunol. 12, 761-769 (2011).

47. Kühn, R., Schwenk, F., Aguet, M. \& Rajewsky, K. Inducible gene targeting in mice. Science 269, 1427-1429 (1995).

48. Oh, H., Siano, B. \& Diamond, S. Neutrophil isolation protocol. J. Vis. Exp. 17, 745 (2008).

49. Pflicke, H. \& Sixt, M. Preformed portals facilitate dendritic cell entry into afferent lymphatic vessels. J. Exp. Med. 206, 2925-2935 (2009). 